Cahiers $d u$ MONDE RUSSE

\section{Cahiers du monde russe}

Russie - Empire russe - Union soviétique et États indépendants

50/2-3 | 2009

L'Europe orientale, 1650-1730. Crises, conflits et renouveau

\title{
L'opposition au pouvoir impérial dans la théorie politique de la Russie ancienne
}

Ses modèles littéraires

Opposition to imperial power in Old Russia's political theory and its literary

models

\section{Ol'ga Novikova-Monterde}

\section{OpenEdition}

Journals

\section{Édition électronique}

URL : https://journals.openedition.org/monderusse/9741

DOI : 10.4000/monderusse. 9741

ISSN : $1777-5388$

Éditeur

Éditions de l'EHESS

\section{Édition imprimée}

Date de publication : 15 septembre 2009

Pagination : 381-394

ISBN : 978-2-7132-2260-3

ISSN : $1252-6576$

Référence électronique

Ol'ga Novikova-Monterde, «L'opposition au pouvoir impérial dans la théorie politique de la Russie ancienne », Cahiers du monde russe [En ligne], 50/2-3 | 2009, mis en ligne le 13 octobre 2012, consulté le 08 septembre 2022. URL : http://journals.openedition.org/monderusse/9741 ; DOI : https://doi.org/ 10.4000/monderusse. 9741

Ce document a été généré automatiquement le 8 septembre 2022.

Tous droits réservés 


\title{
L'opposition au pouvoir impérial dans la théorie politique de la Russie ancienne
}

\author{
Ses modèles littéraires \\ Opposition to imperial power in Old Russia's political theory and its literary \\ models
}

Ol'ga Novikova-Monterde

1 Le chercheur qui s'attelle à un sujet aussi classique pour l'histoire politique occidentale que celui du "droit de résistance» éprouvera de nombreuses difficultés face au matériau russe médiéval. Ce fameux droit, qui semble présent sous différentes formes dans toutes les sociétés européennes occidentales, se dérobe lorsque nous nous transposons dans le contexte russe.

2 Ce type de réalité indiscernable est inhérent à la culture de la Russie ancienne en général. Souvenons-nous seulement de la beauté des icônes, si longtemps restée inaperçue, à laquelle il n'était prêté attention que pour en souligner les nombreux écarts par rapport aux canons de l'art classique; ou encore de l'oubli durable dans lequel fut plongée la littérature russe ancienne, à laquelle aucune valeur artistique n'était accordée.

3 Cette réalité demeurait imperceptible du fait que le langage dans lequel était écrit l'ouvrage, ou peinte l'icône médiévale russe, n'était pas compris. La découverte d'un code artistique, bien que d'origine apparentée, mais foncièrement différent du code occidental européen auquel nous sommes habitués, tira de leur mutisme une peinture et une littérature jusque-là réduites au silence. Grâce à cette découverte, ces dernières purent à nouveau être comparées à leurs analogues occidentaux, non plus cette fois comme une imitation malhabile de ceux-ci, mais comme un système artistique indépendant.

4 La théorie politique médiévale russe est victime d'un phénomène comparable. Nous connaissons assez bien l'ensemble des textes politiques accessibles au lecteur russe 
médiéval, même si, comme nous l'espérons, ce corpus sera encore complété et précisé. Cependant, un pan entier de catégories, habituellement considérées comme indispensables dans la tradition occidentale, soit ne figurent pas parmi ces idées politiques, soit s'expriment sous une forme étonnamment apolitique ${ }^{1}$. Certains estiment que cet état de fait est dû au caractère rudimentaire de la vie politique russe médiévale et, par conséquent, à la non-différenciation entre théorisation morale et théorisation politique à proprement parler. L'« invisibilité » du droit de résistance qui nous intéresse ici pourrait, de la même façon, être expliquée par ce faible degré de développement.

5 Cependant, nous nous risquerons à émettre un autre point de vue : afin de "déceler » les points essentiels de la théorie politique russe médiévale, il nous faut chercher le code dans lequel sont écrits ces textes. Il ne s'agit pas uniquement du travail indispensable consistant à définir des termes isolés et à dégager la spécificité de leur emploi ; il s'agit également, selon nous, d'identifier l'origine des idées qui ont donné naissance à ces termes. Ces textes politiques russes médiévaux nous frappent, en effet, non seulement par la langue inhabituelle dans laquelle ils sont écrits, mais aussi par leur caractère étrangement elliptique et fragmentaire. Ce sont généralement des conclusions sans point de départ, des points de départ sans aboutissement, des allusions sans références. Les auteurs exposent rarement leurs idées de façon cohérente et renvoient le plus souvent à un ensemble de concepts et de représentations faisant autorité. En réintroduisant les énoncés dans leur contexte, en reconstituant le point de départ de certaines conclusions, en identifiant certains modèles, nous serons peut-être à même de déchiffrer le code qui se trouve à l'origine de la théorie politique russe médiévale. Le comportement politique des personnages historiques pourrait alors nous apparaître sous un jour nouveau.

6 Le caractère elliptique des textes politiques ne nous semble pas constituer une faiblesse, mais plutôt relever d'un procédé conscient de l'érudit médiéval russe, dont l'éducation était à base de lectures religieuses. Nombre de ces textes, ceux des Pères de l'Église, notamment, ont vu le jour dans une même aire géographique et à une même époque : ce sont des écrits de la civilisation méditerranéenne de l'Antiquité tardive. Parmi eux, les écrits de saint Jean Chrysostome, incroyablement populaires dans la Russie médiévale, sont ponctués d'allusions à des realia de l'Antiquité méditerranéenne (les portiques, les bœufs, les oliveraies, le temple d'Apollon à Daphné, etc.) qu'aucun des lecteurs russes du XVI ${ }^{\mathrm{e}}$ siècle n'était à même d'observer. Les œuvres de saint Jean Chrysostome évoquaient non seulement des objets inconnus et extraordinaires, mais se référaient de surcroît à une manière de penser à laquelle ces lecteurs étaient étrangers. Saint Jean Chrysostome écrivait en effet pour un cercle bien défini d'auditeurs ayant reçu une éducation grecque classique. Ces derniers étaient familiarisés avec une catégorie bien précise d'idées et de concepts qui leur permettaient de saisir les noms et les citations sous-entendus. À la différence des chrétiens grecs de l'Antiquité tardive, les moines érudits de la Russie médiévale étaient, eux, loin de tout percevoir.

7 Néanmoins, ces moines avaient le plus souvent recours à la «lecture lente", s'interrompant souvent et analysant longuement chaque phrase, ce qui leur permettait de reconstituer, du moins partiellement, une partie des raisonnements qui leur échappaient. Le caractère mystérieux, elliptique, des textes sacrés, pouvait facilement être pris comme modèle. En imitant les textes de référence, ils adoptaient l'ellipse comme procédé stylistique. En d'autres termes, il n'était pas rare que les moines érudits 
de la Russie ancienne construisent leur discours en ne mentionnant qu'un seul élément de la comparaison. C'était alors à l'auditeur de reconstituer, dans son esprit, le second élément. Cette forme d'énonciation (sorte de variante du «moins-procédé (minuspriëm $)^{2} »$ de Lotman) était aussi utilisée dans l'argumentation politique. Pour démontrer la validité de l'idée médiévale, c'était la référence aux textes fondamentaux qui servait de preuve. Le style elliptique permettait évidemment de ne citer qu'une partie de la référence nécessaire (dans l'espoir que "le sage entendra à demi-mot») mais aussi de renvoyer à une référence "composite", constituée de fragments d'autorités. Ce procédé d'énonciation, que nous qualifions de "citation complexe" permet à l'énonciateur, en mettant l'accent sur quelques mots-clefs, de renvoyer l'auditeur (ou le lecteur) à un ensemble d'idées et de représentations connu respectivement d'eux et de lui-même? . La lecture des textes politiques hellénistiques (non pas dans l'original, malheureusement, mais, dans notre cas, dans leur traduction anglaise ou russe) nous a convaincue que c'est dans la tradition extrêmement riche qui va d'Isocrate aux Pères de l'Église orientale, d'Eusèbe de Césarée à Agapet et Photios ${ }^{4}$, qu'il faut chercher le contexte dans lequel trouvent leur source les idées politiques russes médiévales. L'influence d'Agapet sur la vie politique russe a été relevée par de nombreux chercheurs $^{5}$, mais l'influence directe ou indirecte d'autres auteurs peut également être mise en évidence. En Russie, la transposition d'idées politiques essentielles s'est effectuée durant plusieurs siècles par l'intermédiaire de l'Église, c'est-à-dire de la liturgie, de la lecture des psaumes et des homélies de saint Jean Chrysostome, de l'hymne des Chérubins, du Canon d'André de Crète, des recueils d'aphorismes des Pères de l'Église, etc.

Directement ou indirectement, les idées transmises par ces textes émanent d'un courant bien précis de pensée politique, à savoir l'école hellénistique. Si cette thèse se confirme, la mise en parallèle de la théorie politique russe médiévale et de la théorie politique occidentale devra alors être envisagée sous un jour nouveau.

10 L'aspect moralisateur de la pensée hellénistique s'expliquait non pas par l'ignorance de la distinction aristotélicienne entre éthique et politique, mais par le peu de foi qu'elle avait dans l'utilité pratique d'une telle distinction. Convaincue à plusieurs reprises de l'inefficacité du pouvoir démocratique dans la cité, la pensée hellénistique trouva dans la monarchie un système d'organisation, lui semblait-il, plus perfectionné, et s'efforça de fixer des limites non pas externes, mais internes au pouvoir politique. Délaissant Aristote, la pensée hellénistique se tourna vers Agapet.

11 À travers Byzance, la culture médiévale russe est l'héritière de la civilisation hellénistique, ultime aboutissement de la pensée politique grecque. La pensée occidentale, elle, qui n'avait pas expérimenté concrètement la vie de la cité, se tourna, à un moment donné de son évolution, vers Aristote, puis vers la tradition de la République romaine, dans lesquelles elle trouva des clefs pour résoudre les problèmes du pouvoir monarchique ${ }^{6}$. Ainsi, la nouveauté des idées politiques occidentales était fondée sur un pan de culture largement oublié, ou, plus précisément, sur de l'ancien que l'on avait délaissé, tandis que le caractère «archaïque » et "peu évolué » de la Russie médiévale reposait sur le « dernier mot » de la pensée politique grecque.

12 La Russie médiévale s'est initiée à la politique grâce aux textes introduits par l'Église orthodoxe. Les prières, souvent traduites de poètes byzantins, les épîtres des prélats, les divers genres de littérature édifiante, depuis les « instructions » des sages jusqu'aux 
vies de saints, tout cet immense corpus de textes était imprégné sinon de la lettre, du moins de l'esprit des concepts et représentations politiques, artistiques et philosophiques de la pensée chrétienne hellénistique ${ }^{7}$.

13 Joseph (Sanin) de Volokolamsk, écrivain politique et prélat des plus influents du Moyen Âge russe, offre un parfait exemple de cette assimilation profonde de la pensée chrétienne hellénistique. L'influence d'Agapet sur sa conception du pouvoir impérial a en effet été relevée plus d'une fois ${ }^{8}$. Notre tâche consiste ici à analyser la justification qu'il donne du droit à la résistance au pouvoir du tsar. Un court paragraphe de son "Épître à un peintre d'icônes et "discours" sur la vénération des icônes " contient une des réflexions les plus détaillées de la littérature russe médiévale sur ce thème.

En s'appuyant sur des citations du Nouveau Testament, l'auteur présente la nécessité de se soumettre au pouvoir séculier comme une sorte de contrat : le « service » est dû en échange de la "protection " assurée par les autorités9. Mais une telle " soumission " a des limites : « Il convient de les servir par le corps et non par l'esprit, de les honorer comme des rois et non comme Dieu ${ }^{10}$. L'obéissance au tsar "dans la grâce comme dans le châtiment » doit ainsi enseigner la " crainte de Dieu », car le tsar est serviteur de Dieu. Cette sentence concise constitue la conclusion ultime à laquelle est parvenue la pensée byzantine, après des siècles de querelles sur les frontières séparant pouvoirs spirituel et profane.

Plus loin, Joseph rappelle la distinction, déjà présente chez les Pères de l'Église ${ }^{11}$, entre le tsar orthodoxe et le tyran impie :

Si le tsar régnant sur les hommes est dominé par ses passions et ses péchés, par la cupidité et la colère, par la perfidie et l'injustice, l'orgueil et la fureur et, pire que tout, par l'impiété et le blasphème, alors un tel tsar ne peut être le serviteur de Dieu, mais un démon, ni un tsar, mais un tyran.

Cette phrase est probablement une transposition de la définition du roi-tyran par l'un des Pères de l'Église les plus appréciés dans la Russie ancienne, saint Jean Chrysostome : «[Le roi-tyran est] celui qui veut régner sur les hommes tout en assouvissant ses passions : la colère, l'orgueil et la concupiscence [...]», tandis que le roi véritable est celui qui «bride ses passions : la colère, l'envie et la concupiscence, qui se soumet en tout à la loi divine $»^{12}$.

17 Cependant, la liste des critères permettant de définir le tsar-tyran chez Joseph de Volokolamsk ne s'arrête pas là et ne coïncide que partiellement avec celle de saint Jean Chrysostome. Il nous semble qu'il ne s'agit pas tant ici de la citation littérale de telle ou telle autorité, que de l'assimilation de certains concepts, empruntés à tout un ensemble d'œuvres diverses. C'est un exemple de citation complexe dont il a déjà été question. Remarquons au passage que la définition des "passions" par l'higoumène de Volokolamsk est construite à partir d'une série de couples synonymes, dans lesquels la seconde définition vient généralement préciser et renforcer la première, soulignant sa consonance religieuse: passions et péchés, perfidie et injustice, colère et rage, incrédulité et blasphème. Dans la définition de Sanin, le caractère hérétique du tyran-« démon » s'exprime plus fortement que dans le texte du Chrysostome :

Et, dans ce cas, tu n'obéiras point à un tel tsar ou à un tel prince, même s'il te torture et menace de te tuer, car il te conduirait à perdre ton honneur et à faire le mal.

Là-dessus, il met en avant les modèles de résistance au roi-tyran : 
Les prophètes, les apôtres et tous les martyrs furent tués par des rois impies, car ils ne se soumirent point à leurs volontés. l'empereur hérétique Constantin II, qui était arien et persécuta les orthodoxes, par le fait qu'il était mal conseillé, et qu'il se repentit ensuite sur son lit de mort ${ }^{18}$. Il faut remarquer que cet espoir d'un repentir avant la mort n'est pas étranger à Timofeev, qui affirme que condamner les «premiers souverains [légitimes] » est un péché, « car seul 
Dieu peut les juger [...] Lui qui sait tout de tous les hommes, non seulement les choses visibles, mais aussi les choses cachées, Lui qui dévoile les pensées secrètes " ${ }^{19}$

D'autres textes, au contraire, décrivent la façon dont l'évêque a reconnu et confondu sans difficulté le tyran. Chez ce même Théodoret de Cyr, saint Ambroise interdit l'entrée de l'église à Théodose qui reconnaît aussitôt ses crimes et « se retire dans son palais en gémissant, le visage couvert de larmes $\star^{20}$. Chez Sozomène, le conflit se résout de la même façon : l'empereur, accusé de la mort d'innocents, est aussitôt frappé d'un profond repentir. Il se repent publiquement à l'église et renonce, durant sa pénitence, aux marques extérieures de respect dues au souverain ${ }^{21}$. En revanche, chez saint Jean Chrysostome, qui relate une histoire similaire, le dénouement est plus réaliste: l'évêque d'Antioche Babylas, après avoir pris la parole contre Julien l'Apostat, est jeté en prison où il meurt dans les fers ${ }^{22}$.

Il est difficile de savoir quel dénouement espérait obtenir le métropolite Philippe (Kolyčev) lorsqu'il décida, à l'instar des prélats antiques, de dénoncer publiquement les crimes d'Ivan IV, mais la similarité profonde entre le récit qui est fait de cet incident et la confrontation de Babylas et de Julien l'Apostat (le «tyran » préféré de la littérature russe médiévale) aurait impressionné Jurij Lotman, qui aimait à écrire que la vie se construit à partir des modèles littéraires. Les actes d'opposition de Philippe et de Babylas ont la même origine (la mort d'innocents), ils se situent dans un même lieu (une église, en présence d'un grand nombre de chrétiens orthodoxes) et la mesure prise contre le tsar Ivan IV est la même que celle prise par Babylas à l'encontre de Julien l'Apostat: l'excommunication (le refus de la bénédiction, auquel eut recours saint Philippe est une des formes de l'excommunication). La réaction du tyran (que Philippe avait certainement envisagée, compte tenu des précédents existant dans l'histoire de l'Église) est également la même : l'évêque gênant est déposé et jeté en prison. Une autre circonstance rapproche les deux récits : la force toute en retenue du comportement de l'évêque, dont la violente protestation s'exprime dans un langage emprunt d'humilité et de tempérance. Nous pourrions nous écrier avec saint Jean Chrysostome :

L'audace dont il fit preuve ne doit pas seule nous étonner, mais également l'étendue de son audace qui, cependant, n'était point outrée. Telle est la sagesse du Christ: elle ne tolère une ardeur au combat ni insuffisante, ni excessive, et observe en tout la juste mesure. ${ }^{23}$

La « juste mesure » dans le discours de Philippe est elle aussi étonnante. Il évite les offensives et les accusations directes :

Sois miséricordieux, glorieux souverain [...]. Ce que je déplore, ce n'est pas le sang innocent qui coule ni que l'on meure dans de grandes souffrances [...] mais j'éprouve de l'inquiétude pour ton âme unique et immortelle. ${ }^{24}$

V. Kolobkov explique une telle tempérance «par le calcul froid du politique expérimenté $»^{25}$. Sans aller à l'encontre de cette conclusion, nous souhaitons néanmoins suggérer une autre raison possible à cette attitude: quiconque avait lu l'abondante littérature édifiante alors disponible avait assimilé l'idée que la juste mesure est le seul comportement adéquat à adopter face à un tyran :

De plus, saint Babylas aurait pu, s'il l'avait voulu, aller plus loin. Celui qui avait renoncé à la vie (et il n'eût point agi ainsi, s'il ne s'était armé de cette pensée) pouvait sans encombre aller jusqu'au bout, couvrir le roi d'outrages, lui arracher son diadème, le frapper au visage, puisqu'il avait déjà porté la main sur sa poitrine. [...] Ainsi Shiméï outragea violemment le roi David et l'appela assassin (II Samuel, 
XVI, 7) ; cependant, je n'appellerai pas cela hardiesse, mais intempérance de langage, insolence de l'esprit, impétuosité, déraison, tout, sauf hardiesse. Je pense que celui qui souhaite confondre autrui doit tenir son âme le plus éloignée possible de l'insolence, de l'orgueil, et doit montrer sa force uniquement par la nature de ses paroles et de ses actes ${ }^{26}$. similitude entre les actions de saint Babylas et celles de saint Philippe. En effet, les faits relatés dans la vita du métropolite moscovite sont confirmés par d'autres sources ${ }^{27}$ et la confrontation entre Ivan le Terrible et Philippe Kolyčev a probablement eu lieu dans des conditions semblables à celles décrites ici. Par conséquent, nous ne pouvons exclure l'éventualité d'une imitation délibérée, d'autant que, selon les termes de Chrysostome, l'évêque d'Antioche a proposé une sorte de modèle :

Il a, pour ainsi dire, posé certaines règles et lois pour que nous en tirions, même dans d'autres domaines, des préceptes de modération. ${ }^{28}$

C'est encore saint Jean Chrysostome qui compare la dénonciation épiscopale à la démarche du médecin :

C'est pourquoi il n'incisait pas uniquement en surface, afin de ne pas laisser de partie du corps atteinte par la maladie, ni trop en profondeur non plus, pour ne pas nuire davantage à la santé du patient par une entaille excessive mais, proportionnant l'incision à la nature de la maladie, il agissait en médecin exemplaire. ${ }^{29}$

Cette comparaison montre de façon éloquente que le but recherché par l'évêque, en s'opposant au tyran, n'était pas la défense de la liberté ou des droits, mais le salut de son âme et de celle du souverain impie. Pour être juste, nous ajouterons que cette "médecine » n'a pas toujours conduit à une fin aussi tragique pour le guérisseur de l'âme, que celle de Babylas et de Philippe, morts "dans les fers». En 1497, le métropolite Simon, soutenu par le haut clergé, parvint à contraindre Ivan III à faire pénitence publique pour le meurtre de son frère :

Le grand prince [...] se prosterna [...] et demeura longtemps ainsi, par force, devant eux. Le métropolite, l'archevêque et l'évêque l'éprouvèrent longuement et lui firent faire longue pénitence, puis lui accordèrent le pardon en lui enjoignant d'expier désormais ses péchés devant Dieu. ${ }^{30}$

Pour revenir à l'histoire de Babylas, il est une question que l'on peut légitimement se poser : s'il n'est pas étonnant que le métropolite Philippe ait pu s'inspirer des modèles des Pères de l'Église, dans quelle mesure cette idée d'opposition comme forme de martyre chrétien était-elle répandue au sein des laïcs?

Dans l'Histoire du grand prince de Moscou de Kurbskij, nous trouvons quelques exemples d'opposition perçue comme légitime et justifiée et il s'agit d'ailleurs des uniques cas de résistance décrits par le bojarin en fuite ${ }^{31}$. Ainsi, Molčan Mitnev, contraint à s'enivrer lors d'un festin, s'insurgea contre le tsar en ces termes:

Ô tsar ! En vérité, tu nous obliges, comme toi, à boire cette boisson maudite, mêlée au sang des vrais chrétiens, nos frères. ${ }^{32}$

De même, lors d'une orgie, le prince Repnin refusa d'obéir à l'ordre du tsar qui voulait le faire danser masqué :

Voyant cette conduite licencieuse, cet homme noble et respecté se mit à pleurer et lui dit qu'«agir ainsi n'était pas digne d'un tsar chrétien ». Le tsar voulut alors l'y contraindre et lui dit: «Amuse-toi et participe à nos jeux! ", tout en prenant un masque qu'il posa sur son visage. Repnin rejeta le masque, le foula aux pieds et 
dit : « Ne me contrains pas, moi qui fais partie de ton conseil, à cette folie et à cette indécence ! 33

Vasilij Kalugin a mis en évidence la logique interne qui préside à la construction de l'image du roi-tyran dans l'Histoire du grand prince de Moscou de Kurbskij, à la lumière de l'Exposé de la foi orthodoxe de saint Jean Damascène ${ }^{34}$. La violence des accusations de Mitnev et de Repnin requiert, elle aussi, une explication car, de notre point de vue actuel, l'ivrognerie et les bouffonneries, comportements affligeants en soi, ne peuvent constituer des critères de définition du tyran. Nous devons, pour l'expliquer, adopter un nouveau point de vue qui révélera une dernière facette du roi impie. Il nous semble que la réponse se trouve dans un autre discours de saint Jean Chrysostome, que Kurbskij cite ailleurs dans son Histoire. Pourquoi donc Kurbskij, admirateur et traducteur de saint Jean Chrysostome, estimait-il que les exemples de Mitnev et de Repnin convaincraient le lecteur qu'Ivan IV était, non pas « le souverain par la grâce de Dieu de son propre pays dès l'origine ${ }^{35}$ » (son « seigneur naturel ${ }^{36}$ », en somme), « mais un démon, un tyran»?

Selon saint Jean Chrysostome, saint Babylas s'opposa à l'un des principaux archétypes de «tyran»: Julien l'Apostat. Un autre exemple classique de roi impie était le roi Hérode de l'Évangile et Kurbskij cite à ce propos le Discours sur Hérode de saint Jean Chrysostome: «[...] tyran de ses cités, bourreau de ses propres guerriers [...], destructeur de ses amis $»^{37}$. Le lecteur de l'Homélie sur la Pénitence, sur Hérode et sur JeanBaptiste, apprenait que les festins où l'on boit et où l'on danse mettent l'âme du chrétien en péril et ruinent les royaumes. Le roi qui ne se préoccupe pas du bien-être du peuple orthodoxe qui lui a été confié, est un "vil apostat ", selon l'expression de Joseph de Volokolamsk, et ne peut être autre chose qu'un tyran.

Et pour que tu saches que les spectacles indécents et menant à la ruine des âmes, que l'on tolère lors des festins, méritent d'être blâmés, je te rappellerai le festin du roi, ou plus exactement, du tyran, qui est décrit dans le livre des Évangiles. Un repas était donné chez le roi ou, plus exactement, chez le tyran, pour l'anniversaire d'Hérode, renard abominable, bête rusée, entachée de sang; connaissant son caractère, le Seigneur lui-même ne dit point : «Allez dire à ce lion » (bien que cet animal représente le roi), mais «Allez dire à ce renard » (Luc, XIII, 32), car, bien qu'il eût la force du lion, de caractère, il ressemblait au renard. Quand le festin commença et que tout le monde fut rassemblé pour boire le vin, une danseuse vint se joindre à la fête. Ce spectacle eût déjà été honteux, indigne et lamentable, si elle s'était montrée sur la scène d'un théâtre, mais combien pire au festin d'un roi ? ${ }^{38}$

Il faut se souvenir ici que dans le système de valeurs chrétien hellénistique, la mesure était considérée comme une qualité politique, et ce n'est pas un hasard si un recueil russe de modèles de gouvernement et de justice équitables du XIV ${ }^{e}$ siècle s'intitulait $L a$ juste mesure. C'est ainsi que Timofeev reprochait aux usurpateurs Boris Godunov et Otrep'ev ${ }^{39}$ d'ignorer toute mesure, d'allouer des récompenses disproportionnées, « dignes des excès d'Hérode ». Après avoir décrit comment Hérode, enivré par le vin et avide de plaisirs, enfreint les lois divines et le sens de la mesure, en promettant une récompense immodérée à la danseuse, saint Jean Chrysostome souligne le caractère néfaste des festins pour les royaumes et pour les rois :

Vois-tu combien la soif de plaisir rabaisse la dignité des rois jusqu'à terre ? Vois-tu combien elle avilit le royaume? «Tout ce que tu demandes, je te le donnerai, même jusqu'à la moitié du royaume. » Que vaut, à ton avis, sa dignité royale ? As-tu perdu la vue ? Il suffit à cette jeune fille de danser pour que tu lui en accordes une moitié ; mais si elle avait dansé une seconde fois, le roi ne serait plus qu'un homme du commun. Où est la dignité de ceux qui s'adonnent aux plaisirs? Où est la pourpre

Cahiers du monde russe, 50/2-3 | 2009 
des dépravés? Il a jeté la moitié du royaume aux pieds d'une danseuse; prie le ciel qu'elle ne danse pas une seconde fois, ou que, si elle danse encore, elle ne danse pas aussi bien. Sinon tu n'auras plus qu'à mendier. Ainsi, rien ne justifie la récompense allouée ; qu'exigeait donc cette danseuse ${ }^{40}$ ?

Kurbskij écrivait pour des lecteurs capables de reconstituer sans difficulté le réseau d'associations reliant la danse du festin à la figure funeste d'Hérode, le bourreau des innocents. L'existence d'un tel lien trouve une confirmation chez Timofeev, de même que le penchant pour les festins débridés et les danses, qui entrait dans la liste des traits du «tyran classique». Ainsi, dans sa parabole Du veuvage de la Moscovie, les autorités corrompues sont accusées, entre autres, d'organiser des festins somptueux et de « ruiner le pays tout entier $»^{41}$. adversaire couronné était un tsar-tyran, voire l'Antéchrist décrit en détail par saint Jean Damascène. Avec l'Histoire, véritable martyrologe où figurent les nouveaux martyrs russes, suppliciés par ce "mangeur de chair humaine", et ses nombreuses traductions des vies de martyrs, Kurbskij tenait sa promesse, faite dans sa première Épître à Ivan IV : «jusqu'à la fin de mes jours, je ne cesserai de crier avec des larmes vers Toi, ô Trinité plus qu'éternelle ${ }^{42}$. ». Ces écrits constituent, avec les lettres à Ivan le Terrible, l'une des plus éclatantes manifestations de résistance et de dénonciation de la tyrannie.

Ce modèle d'opposition se résolvant en une condamnation verbale, légitimé par la théorie politique de la Russie ancienne, resta longtemps en usage malgré son manque flagrant d'efficacité (de notre point de vue). Une génération plus tard, durant le Temps des Troubles, l'auteur du Récit nouveau du très glorieux royaume de Russie décrivait encore l'opposition du patriarche Hermogène selon ce même modèle du saint qui invective le souverain impie :

Que peut-il y avoir de plus digne d'éloges, de plus extraordinaire, et de plus intrépide? Se trouver entre les mains de son ennemi juré, aux portes de la mort, manquer de tout, et cependant ne pas détourner son visage de l'adversaire, et lui dire en face qu'il n'arrivera pas à ses fins [...]

41 Un passage du Récit nouveau nous aide à comprendre la valeur transcendantale qu'on attribuait à ce genre de joute verbale :

Ici, parmi nous, ce pilier inébranlable dont j'ai parlé se dresse, solide, sans broncher, ferme dans son esprit. Ce ne sont pas seulement les murailles de notre grande cité qu'il tient à bout de bras : il soutient et instruit tous ceux qui l'habitent et les empêche de sombrer dans le précipice. Par ses paroles, il apaise et dompte cette immense mer humaine. Vous le voyez vous-mêmes. Et s'il n'était pas là, le prince de l'Église, qui se serait dressé contre nos ennemis, qui leur aurait tenu tête? Il y a longtemps que, habités par la peur, nous aurions enfreint les commandements de Dieu, longtemps que nos âmes auraient perdu courage, et nous notre salut. ${ }^{43}$

Nous n'avons bien sûr étudié ici qu'une seule forme de résistance au tsar-tyran, mais il s'agit de celle que la théorie politique de l'ancienne Russie, héritière de la tradition byzantine et hellénistique, considérait comme légitime et louable. Il existait en réalité d'autres formes d'opposition, maintes fois décrites dans les travaux des historiens: déposer des pétitions collectives, se porter garants des condamnés, refuser de servir le tsar, ce qui impliquait la fuite en Lituanie ou la tonsure. Cependant, personne, dans la Russie ancienne, n'aurait osé se décrire comme résistant au pouvoir légitime du tsar. C'est pourquoi les actes de protestation et d'opposition, en général, y compris l'opposition verbale considérée comme admissible, s'exprimaient dans le langage de la 
soumission. Tous reconnaissaient les Rurikides comme le lignage des tsars élus de Dieu. Même la forme la plus extrême d'opposition, à savoir le complot, ne visait qu'à mettre sur le trône un autre membre de la dynastie, dont les prétentions étaient «mieux fondées » : l'apparence de la soumission au pouvoir légitime était ainsi préservée.

Cependant, les formes non verbales d'opposition n'étant consacrées ni par l'autorité des Pères de l'Église, ni par la littérature édifiante, trouvent donc peu d'échos dans les œuvres littéraires du Moyen Âge russe.

Le fait que de nombreux contemporains d'Ivan le Terrible, voire même leurs descendants, aient eu recours à d'anciens modèles proposés par la littérature édifiante pour choisir leur ligne de conduite politique ne doit en aucun cas nous surprendre. En effet, de tout temps l'homme a cherché dans les livres ou dans la tradition orale des modèles à suivre et des héros de qui s'inspirer pour faire face à des situations hors du commun.

(traduit du russe par Diane Chertier-Vitovtov)

\section{NOTES}

1. Soulignons l'apparition, ces dernières années, d'une littérature abondante traitant des aspects les plus divers des relations de pouvoir entre le tsar et les autres acteurs du jeu politique : la noblesse et les hiérarques de l'Église. Sans en faire un recensement exhaustif, nous ne citerons que quelques travaux, directement liés à nos " héros": K.Ju. Erusalimskij, "Ideal'nyj sovet v Istorii o velikom knjaze Moskovskom » [Le conseil idéal dans l'Histoire du grand prince de Moscou], in Tekst $v$ gumanitarnom znanii : Materialy mežvuzovskoj naučnoj konferencii [Le texte dans les sciences humaines: Actes du colloque interuniversitaire], M., 1997; du même auteur, «Predstavlenija Andreja Mihajloviča Kurbskogo o knjažeskoj vlasti i russkih knjaz'jah IX-serediny XVI veka » [Conception du pouvoir princier et des princes russes $d u \mathrm{IX}^{\mathrm{e}}$ au milieu du $\mathrm{XVI}^{\mathrm{e}}$ siècle chez Andrej Mihajlovič Kurbskij], Socium. Al'monah social'noj istorij, 4, p. 71-100; du même auteur " "Izmennym obyčaem" : Livonskaja vojna i predstavlenija o gosudarstvennoj izmene v Rossii » [ "Agissant en traître »: la guerre de Livonie et la conception de la haute trahison en Russie], ibidem, 6, p.61-84; A.V. Karavaškin, Russkaja srednevekovaja publicistika [La littérature politique dans la Russie médiévale], M. : Prometej, 2000 ; Jaakko Lehtovirta, Ivan IV as Emperor: The Imperial Theme in the Establishment of Muscovite Tsardom, Painosalama, 1999; Sergej N. Bogatyrev, The Sovereign and his Counsellors. Ritualised Consultations in Muscovite Political Culture, Helsinki : The Finnish Academy of Science and Letters, 2000 ; Isabel de Madariaga, Ivan the Terrible, Londres-New Haven: Yale University Press, 2005.

2. Ce terme est introduit pour la première fois par Ju.M. Lotman dans son livre Analiz poetičeskogo teksta [Analyse du texte poétique], M. : Učpedgiz, 1972.

3. Aleksandr I. Filjuškin appelle «thésaurus » ce corpus d'idées et de représentations de référence. Voir A.I. Filjuškin, Andrej Mihajlovič Kurbskij. Prosopografičeskoe issledovanie i 
germenevtičeskij kommentarij $k$ poslanijam Andreja Kurbskogo Ivanu Groznomu [Andrej Mihajlovič Kurbskij. Étude prosopographique et commentaire herméneutique des lettres de Kurbskij à Ivan le Terrible], SPb. : éd. de l'Université de Saint-Pétersbourg, 2007.

4. Le cadre du présent article ne permet pas de fournir suffisamment d'arguments pour étayer de façon détaillée cette affirmation. Des illustrations précises en faveur de cette théorie seront exposées plus loin, mais il faudrait pour la justifier lui consacrer une étude distincte. Il reste à espérer que ce travail sera un jour effectué par des chercheurs capables d'étudier les textes hellénistiques dans l'original.

5. Voir, par exemple, Vladimir E. Val'denberg, « Nastavlenie pisatelja IV veka Agapita v russkoj pis'mennosti » [Le sermon d'Agapet, écrivain du Iv siècle, dans la littérature russe], in Vizantijskaja civilizacija $v$ osveščenii rossijskih učenyh [La civilisation byzantine du point de vue des chercheurs russes], M.: Ladomir, 1999, p. 32-40; I. Ševčenko, "A Neglected Byzantine Source of Muscovite Political Ideology ", Harvard Slavic Studies, II, 1954 ; Jakov S. Lur'e, Ideologičeskaja bor'ba v russkoj publicistike konca XV-načala XVI veka [Le combat idéologique dans la littérature politique russe de la fin $d u \mathrm{XV}^{e}$ et du début du XVI siècle] M.-L. : AN SSSR 1960.

6. Il est intéressant de constater qu'en Occident, l'intérêt porté à Agapet s'accrut à la Renaissance, quand apparurent les théoriciens de la monarchie absolue. Aristote perdit, lui, de son attrait. Voir l'article du byzantiniste Antonio Bravo Garcia sur deux nouvelles traductions d'Agapet, apparues durant le Siècle d'or espagnol, «Dos traducciones de Agapeto impresas en el siglo XVI ", Revista de Filologia Romanica, 2, 1985, p. 225-232.

7. Parmi les nombreux exemples existants, nous n'en citerons que quelques-uns : on trouve une série d'emprunts importants à Agapet dans l'Histoire de Barlaam et de Josaphat ou dans l'épître de Joseph de Volokolamsk à Basile III. Dans la stichère [cantique de louanges], écrite par la poétesse byzantine Kasia, qui est toujours chantée à l'époque de Noël dans les églises orthodoxes, le pouvoir de l'empereur est comparé à celui du Christ: « La polyarchie (polyarchia) laissa place au règne unique d'Auguste sur la terre : de même, quand Tu naquis homme de la Très Pure, le polythéisme (polytheia) des idoles disparut. Les cités de ce monde (polis) se réunirent sous le sceptre (basileia) d'un seul: de même, les nations (ethnia) crurent au règne d'un Dieu unique. Les hommes se sont soumis au recensement sur l'ordre de César: de même nous autres croyants nous comptons au nombre de Tes fidèles, en Ton nom, Toi notre Dieu qui T'es fait homme. Grande est Ta miséricorde, gloire à Toi, Seigneur. » Les termes grecs sont cités (au nominatif singulier) d'après Raffaele Cantarella, éd., Poeti bizantini (testo greco a fronte), vol. II, Milan : Rizzoli, 2000, p. 626-627. Il ne faut pas perdre de vue non plus la signification des idées politiques développées dans les psaumes ni les commentaires patristiques qui s'y rapportent.

8. Val'denberg, «Le sermon d'Agapet ...», p. 33 ; Ševčenko, «A Neglected Byzantine Source... », p. 178.

9. Citations d'après "Poslanija ikonopiscu i "slova" o počitanii ikon" [Épître à un peintre d'icônes et « discours » sur la vénération des icônes], dans Natal'ja A. Kazakova et Jakov S. Lur'e, Antifeodal'nye eretičeskie dviženija na Rusi [Les mouvements hérétiques antiféodaux de la Russie ancienne], M.-L. : AN SSSR, 1955, p. 346.

10. Cette affirmation est également la transposition d'un passage des Évangiles: «Esclaves, obéissez à vos maîtres d'ici-bas avec crainte et tremblement, d'un cœur 
simple, comme au Christ, non parce que l'on vous surveille, comme si vous cherchiez à plaire aux hommes, mais comme des esclaves du Christ qui s'empressent de faire la volonté de Dieu » (Épître aux Éphésiens, 6, 5-6). Plus haut, l'auteur cite librement les passages suivants: «Tu ne maudiras pas le chef de ton peuple» (Actes des Apôtres, XXIII, 5) ; « Craignez Dieu et honorez le roi » (I Épître de Pierre, II, 17) ; « Rendez à César ce qui est à César, et à Dieu ce qui est à Dieu » (Marc, XII, 17 ; Luc XX, 25).

11. Saint Jean Chrysostome, Homilia in principium Proverbiorum, 2, PG, 31; col. 389 ; Egloga de imperio, Homélie XXI, PG, 63, col. 605 ; Comparatio regis et monarchi, PG, 47, col.388 sq. Citations d'après Francis F. Dvornik, Early Christian and Byzantine Political Philosophy, Washington: The Dumbarton Oaks Center for Byzantine Studies, 1966, p.693-695. Basile de Césarée, Grégoire de Nysse et Théodoret de Cyr ont également écrit à propos du roi-tyran et du roi juste, $c f$. Dvornik, ibid., p. 690-691, 792-794.

12. Dvornik, ibid., p. 695.

13. Nous trouvons ce point de vue chez Théodoret de Cyr, par exemple, $c f$. Dvornik, Early Christian, p. 794, et dans les Menées du métropolite Macaire : «Slovo pohval'noe L 'va filologa Mihailu i Feodory Černigovskim » [Panégyrique de Michel et Théodore de Černigov par Léon le Philologue], Pamjatniki literatury Drevnej Rusi. Vtoraja polovina XVI veka [Monuments littéraires de l'ancienne Russie, seconde moitié du XVI ${ }^{e}$ siècle], M.: Hudožestvennaja literatura, 1986, p. 486-488.

14. Perepiska Ivana Groznogo s Andreem Kurbskim [Correspondance entre Ivan le Terrible et Andrej Kurbskij], M. : Nauka, 1981, p. 65.

15. Vremennik Ivana Timofeeva [Chronique d'Ivan Timofeev], SPb.: Nauka, 2004, p. 158 et 11.

16. Maksim Grek, "Slovo pečal'lnoe... » [Maxime le Grec, "Lamentation ... »] in Krasnorečie Drevnej Rusi [L'éloquence de la Russie ancienne], M. : Sovetskaja Rossija, 1987, p. 221-225.

17. Vremennik Ivana Timofeeva, p. 13-14.

18. Voir Dvornik, Early Christian, p. 790.

19. Vremennik Ivana Timofeeva, p. 33.

20. Voir Dvornik, Early Christian, p. 791.

21. Ibidem.

22. Saint Jean Chrysostome, "Slovo o blažennom Vavile, a takže protiv Juliana, i k jazyčnikam » [Discours sur le bienheureux Babylas, ainsi que contre Julien et adressé aux païens], in Polnoe sobranie sočinenij [ [Euvres complètes], t. II, livre II, $\mathrm{n}^{\circ} 11$, M. : Ispovednik. Nous avons consulté la version électronique : http://www.ispovednik.ru/ zlatoust

23. Ibidem.

24. La vie de saint Philippe dans la rédaction de Kolyčev. Citation d'après Vladimir A. Kolobkov, Mitropolit Filipp i stanovlenie moskovskogo samoderžavija [Le métropolite Philippe et la genèse de l'autocratie moscovite], SPb. : Aleteja, 2004, p. 613.

25. Ibidem, p. 302.

26. Saint Jean Chrysostome, « Slovo o blažennom Vavile... ».

27. Kolobkov, Mitropolit Filipp, p. 341, note 236.

28. Ibidem. 
29. Saint Jean Chrysostome, "Slovo o blažennom Vavile... ». Rappelons que dans les Ménées de Macaire, le Christ (auquel pouvait aisément être comparé l'évêque, si l'on transposait son exemple dans la réalité terrestre) agit également comme un médecin : "Ide ubo i vračem sie ljubezno : egda bo bolezn' napoen'mi i objazaniem povinuti ne v "zmogut', togda rezania i žženia prilagaiut, v eže pol'zy podati stradaiuščemu » [Les médecins ont coutume d'agir ainsi : lorsqu'ils ne peuvent vaincre la maladie ni par les médicaments, ni par des pansements, alors ils ont recours aux cautères et aux incisions, qui sont bénéfiques au malade], «Slovo pohval'noe... », p. 488.

30. PSRL, 24, p. 214.

31. Ce sont les seuls cas rapportés par Kurbskij qu'on puisse qualifier d'actes d'opposition réelle au tyran. Dans les autres cas, il s'agit de victimes innocentes immolées à l'exemple du Christ (Kurbskij fait dire au jeune prince de Suzdal', qui vient de ramasser la tête coupée de son père : «Blagodarju tja, $O$ carju vekom [...] iže spodobil esi nas" nepovinym ubiennym byti, jako i sam ot bogobornyh židov zaklan" esi » [Je te remercie, ô Souverain éternel, [...] de nous avoir jugés dignes d'être tués, nous autres innocents, de même que Toi, l'Agneau innocent, Tu fus tué par les juifs impies], «Istorija o velikom knjaze Moskovskom » [Histoire du Grand Prince de Moscou], in Monuments littéraires de l'ancienne Russie, seconde moitié du Xvie siècle, p. 332 [trad. anglaise par J.L.I. Fennell, Prince A.M. Kurbsky's History of Ivan IV, Cambridge, 1965 ; trad. française par M. Forstetter, A.V. Soloviev, Histoire du règne de Jean IV (Ivan le Terrible), Genève, 1965]. Dans ce passage, l'identification des victimes d'Ivan le Terrible au Christ, et celle d'Ivan le Terrible lui-même aux bourreaux impies apparaissent de façon particulièrement nette.

32. Kurbskij, «Istorija o velikom knjaze Moskovskom », p. 394.

33. Ibidem, p. 328.

34. Vasilij Vasil'evič Kalugin, Andrej Kurbskij i Ivan Groznyj [Andrej Kurbskij et Ivan le Terrible], M. : Jazyki Russkoj kul'tury, 1998, p. 177 sqq.

35. «Iz zapisej peregovorov russkogo pravitel'stva s poslom imperatora Svjaččennoj Rimskoj Imperii » [Extraits des procès-verbaux des pourparlers entre le gouvernement russe et l'ambassadeur de l'empereur du Saint Empire romain] in L'idea di Roma a Mosca, Rome : Herder, 1989, p. 6.

36. En français dans le texte.

37. Kurbskij, «Istorija o velikom knjaze Moskovskom », p. 322.

38. Saint Jean Chrysostome, "Slovo o Pokajanii, i ob Irode i ob Ioanne Krestitele" [Homélie sur la Pénitence, sur Hérode et sur Jean-Baptiste], in Polnoe Sobranie sočinenij [Cuvres complètes], t. VIII, livre II, M.: Ispovednik. Version électronique: www.ispovednik.ru/zlatoust/Z08_2/t08_2.htm

39. Vremennik Ivana Timofeeva, p. 40 et 156.

40. Saint Jean Chrysostome, « Slovo o Pokajanii, i ob Irode... »

41. Vremennik Ivana Timofeeva, p. 156.

42. Perepiska Ivana Groznogo s Andreem Kurbskim, p. 10.

43. Nadežda F. Droblenkova, Novaja povest' o preslavnom Rossijskom carstve i sovremennaja ej agitacionnaja patriotičeskaja pis'mennost' [Le Récit nouveau du très glorieux royaume de Russie et la propagande patriotique de son temps] M.-L. : AN SSSR, 1960, p. 195, 196-197. 


\section{RÉSUMÉS}

Résumé

Pourquoi les textes politiques importants de l'ancienne Russie adoptent-ils un ton moralisateur? Pourquoi sont-ils si souvent fragmentaires, inachevés? Pourquoi ne trouve-t-on pas, dans la pensée politique de l'empire des tsars, certaines idées essentielles, présentes chez presque toutes les monarchies d'Europe?

Il arrive que l'on explique ces particularités par le caractère arriéré de la société russe. L'auteur propose une autre interprétation: la pensée russe et celle d'Occident s'inspirent de sources différentes, quoique apparentées.

Alors que l'Occident prend comme point de départ la théorie aristotélicienne de la polis, la doctrine russe sur la monarchie suit la tradition hellénistique tardive, transmise par les théologiens de l'Église orientale. La prédilection pour le style fragmentaire ferait partie, selon l'auteur, d'une technique rhétorique délibérée, qui s'efforce d'imiter les textes fondateurs de la patrologie orientale, notamment par l'emploi d'une forme particulière de citation (désignée ici par l'expression « citation globale»).

S'agissant du droit de résister au monarque, l'auteur conclut qu'à la fin $d u \mathrm{XVI}^{\mathrm{e}}$ et au début du $\mathrm{XVII}^{\mathrm{e}}$ siècle, certains théoriciens russes, et même des dirigeants politiques, voyaient dans les récits littéraires d'affrontement entre le tyran et le héros chrétien, tels qu'on les trouve chez saint Jean Chrysostome et autres auteurs des premiers temps de l'Église, le parfait exemple de la conduite à tenir face à un monarque impie. Il en résulte que le droit de résistance était à leurs yeux un devoir du croyant comme individu, non la prérogative d'un groupe politique quelconque. Des cas de résistance collective sont certes historiquement attestés, mais pour être considérée comme légitime, la résistance devait être individuelle et non-violente.

Opposition to imperial power in Old Russia's political theory and its literary models Abstract

Why are medieval Russia's important political texts written in moralizing terms? Why do so many of them seem fragmentary, unfinished, to us? Why is it impossible to find in the political thought of the Russian Empire essential ideas that existed in almost all European monarchies?

This situation is sometimes explained by the backwardness of Russian society. The author offers a different explanation: the difference between Russian and Western political thoughts is due to the fact that they sprang from different, though related, sources.

While the origin of Western political thought was Aristotle's theory of polis, Russian doctrine about monarchy comes from the later Hellenistic tradition, transmitted through the theology of the Eastern Church. The fragmentary style of Russian political thinkers was, the author suggests, a deliberate rhetorical device. This rhetorical strategy and its related special form of quotation (called here "global quotation") were meant to imitate the founding texts of Oriental Fathers.

Analysis of the right to resist royal power leads to the conclusion that in the late sixteenth and early seventeenth centuries, some Russian political thinkers, and even what we might call "political leaders," perceived the literary examples of spiritual confrontation between the tyrant and the Christian hero, as described in the works of Saint John Chrysostom and other early Christian writers, as the archetypical way of dealing with an unrighteous monarch. This implies that the right to resist the tyrant was interpreted as the believer's individual obligation rather than the prerogative of political groups. The author does not deny cases of collective resistance, but points out that only a certain type of individual and non-violent resistance was legitimated by Russian political thought. 
AUTEUR

OL'GA NOVIKOVA-MONTERDE

Universidad Autónoma de Madrid, Departamento de Ciencias Politicas 